\title{
Miranda
}

Revue pluridisciplinaire du monde anglophone /

Multidisciplinary peer-reviewed journal on the English-

speaking world

16 | 2018

L'expérimental dans la littérature et les arts

contemporains

\section{Simon Starling, A l'ombre du pin tordu}

MRAC, 4/11/2017 - 18/03/2018

\section{Bénédicte Coste}

\section{(2) OpenEdition}

\section{Journals}

Édition électronique

URL : http://journals.openedition.org/miranda/11776

DOI : 10.4000/miranda. 11776

ISSN : 2108-6559

Éditeur

Université Toulouse - Jean Jaurès

\section{Référence électronique}

Bénédicte Coste, "Simon Starling, A l'ombre du pin tordu », Miranda [En ligne], 16 | 2018, mis en ligne le 06 juin 2018, consulté le 16 février 2021. URL : http://journals.openedition.org/miranda/11776 ; DOI : https://doi.org/10.4000/miranda.11776

Ce document a été généré automatiquement le 16 février 2021.

\section{(c) $(1)$}

Miranda is licensed under a Creative Commons Attribution-NonCommercial-NoDerivatives 4.0 International License. 


\section{Simon Starling, A l'ombre du pin tordu}

MRAC, 4/11/2017 - 18/03/2018

\section{Bénédicte Coste}

1 L'exposition consacrée à l'artiste britannique Simon Starling² (1967 - ) à Sérignan témoigne exemplairement du tournant pris depuis les œuvres des années 1990 et 2000 dont l'une, Shedboatshed, lui a valu le prix Turner en 2005. À vrai dire, l'inflexion de son art vers une interrogation portant non sur l'identité, mais sur la présence était sensible dans une précédente exposition dédoublée entre le Mac/Val et le Parc Saint Léger en 2009.

2 Pratiques de la déconstruction et de la reconstruction, les premières propositions de l'artiste conceptuel portaient sur la nature de l'objet. A Charles Eames 'Aluminium Group' Chair Remade Using the Metal from a 'Marin Sauselito' Bicycle / A 'Marin Sausalito' Bicycle Remade Using the Metal from a Charles Eames 'Aluminium Group Chair' (1997 autrement appelé Work, Made-ready, présenté à la Kunsthalle de Berne) utilisait le métal d'une chaise pour créer un vélo et le métal d'un vélo pour créer une chaise. L'objet se fait, se défait et se refait : tout peut toujours se transformer, affirme Starling commentant son travail du matériau - « My work is about material on a very fundamental level about stuff, about atoms $»^{3}$ - pour demander à son spectateur de se méfier du visible, et des apparentes différences entre les objets.

Carbon (Pedersen) hybride deux bicyclettes Pedersen, ancêtre de la motocyclette (1897), pour qu'elles puissent être actionnées par des tronçonneuses. Les éléments de la bicyclette peuvent alors être séparés pour créer deux tabourets, une tente et une broche de cuisson. Les objets se transmutent, se fondent, se séparent et se recomposent dans une série d'œuvres dont nous ne présentons ici que quelques-unes. L'importance de l'expérimentation se noue à la question du déplacement, cette fois de l'artiste vivant et travaillant à Copenhague et Berlin, pour s'incarner dans un trajet en Volvo de l'Écosse vers l'Espagne, destiné à rapporter des rhododendrons, plante autrefois importée d'Espagne en Écosse où elle avait proliféré au risque de la flore locale (Rescued Rhododendrons 2000). Flaga (1972-2000) est le trajet d'une Fiat 126 de Pologne, où certaines de ses pièces ont été changées puisque c'est là que se fabrique désormais le modèle, en Italie avant que la voiture finisse accrochée au mur d'une galerie peinte aux 
couleurs du drapeau polonais. La question du déplacement des hommes et des objets se pose plus vivement lorsque Starling s'interroge sur sa propre condition d'artiste et affiche une préoccupation marquée pour l'écologie au début des années 2000. Shedboatshed (Mobile Architecture $n^{\circ}$ 2) (2005), cabane trouvée le long du Rhin, est transformée en barque et amenée à Bâle, puis retransformée en cabane et exposée, tandis que l'artiste déclare: "My work thinks out loud about what it means to constantly travel as an artist. $»^{4}$

4 Starling s'intéresse ensuite à la question de l'original et de la copie : un monolithe d'une tonne se voit transporté d'Avonmouth au port de Bristol via la marée. Les formes scannées du monolithe sont ensuite reproduites à l'aide d'une fraiseuse avant que la pierre et son double soient exposés ensemble (Rockcraft 2008). L'intérêt de Starling pour la technologie croise son questionnement de l'utopie dans le projet suivant: Autoxylopyrocyclobotos (2007). Le Loch Long (barque) repêché au fond du lac Windermere est transformé en bateau à vapeur dont la chaudière est alimentée par sa propre coque. À travers cette embarcation vouée à sombrer à l'embouchure de la Clyde à Glasgow, région durement frappée par la désindustrialisation, Starling et son équipier réfutent in vivo et in aqua l'utopie du moteur perpétuel sourdement à l'œuvre dans l'exploitation forcenée des ressources naturelles.

5 À cette époque, Starling a un projet de plus grande ampleur mêlant installations, récit, sculptures, éditions, documents filmiques et/ou visuels, qui va se réaliser en deux lieux, le Mac/Val et le Parc Saint Léger, deux épisodes autonomes quoique pensés ensemble, illustrant là encore la dé/re/composition du matériau, la mise en scène d'objets et la publication/documentation de son activité. Thereherethenthere ${ }^{5}$ (2009) se déploie ainsi à Pougues-les-Eaux avec "La Source", où, sur le site d'une ancienne usine d'embouteillage, Starling agrandit la photo du stockage des bouteilles trouvée sur les lieux jusqu'à sa pixellisation, avant d'en découper une portion dont il replace chaque point dans l'espace d'exposition sous la forme de boules de verre noir soufflées au CIAV de Meisenthal. L'image trouvée par hasard se métamorphose en installation sculpturale fonctionnant comme hommage à l'activité disparue.

$6 \mathrm{Au} \mathrm{Mac} / \mathrm{Val}$, « CEuvres 1997-2009» est une rétrospective d'une dizaine d'œuvres empreintes d'humour, de gravité et de poésie fonctionnant comme une histoire des formes et une exploration de l'histoire de la fabrication et de la réception de l'œuvre, sous l'angle de la narrativité. L'exposition inclut :

7 Les Maquettes en blanc (1995-2009), soient les livres publiés par Starling durant quatorze ans, pris comme partie intégrante de l'œuvre sous la forme d'un livre blanc ;

8 Rockcraft $^{6}$ (2008) ;

Three White Desks ${ }^{7}$ (2008-2009)

Flaga (1972-2002) ;

D1-Z1 $(22,686,575: 1)^{8}(2009)$;

Work, Made-Ready, Kunsthalle Bern (1997)

Exposition $^{9}$ (2004) ;

Silver Particle/Bronze (After Henry Moore) ${ }^{10}$ (2008).

Mirrored Wall Head ${ }^{11}$ (2008).

9 Réécriture d'Eboshi-Ori, Project for a Masquerade (Hiroshima, 2011) montre la fascination de l'artiste pour les récits: il s'agit d'une installation mettant en scène neuf personnages célèbres de la guerre froide, un film et une proposition de représentation théâtrale tout en incorporant l'histoire d'une œuvre de Moore existant elle-même sous 
différentes réalisations et noms (Nuclear Energy, grand monument à Chicago de 1967 et Atom Piece de 1964-5, petite sculpture de bronze acquise par le musée d'Hiroshima en 1987). Starling pose de nouveau la question du visible et de la nature intrinsèque de l'objet : comment savons-nous qu'un objet est ce qu'il apparait ? En outre, ce qu'il faut bien qualifier de réécriture artistique d'œuvres consacrées, tant les métamorphoses de Starling possèdent une forte dimension narrative, se double d'une méditation sur l'œuvre.

10 Autre pierre de touche sur le chemin menant à l'exposition présentée à Sérignan : Home-Made Eames (Formers, Jigs and Molds) (2002) recréant sous forme d'œuvre d'art une chaise en fibre de verre Eames (1948), laquelle était à l'origine un objet de consommation de masse et la première chaise à être fabriquée à la chaîne. En parallèle, Starling documente les étapes de son travail au moyen de quatre photos à tirage chromogène. Cette installation annonce les œuvres exposées à Sérignan où l'artiste propose quatre œuvres en dialogue afin de méditer sur l'expérimentation artistique (décomposition, transformation de l'objet, réécritures multimédias) tout en invitant son spectateur à interroger son rapport au visible. Prise à Portiragnes, la photo initiale du pin renvoie à son utilisation comme motif décoratif dans le théatre Nô ainsi qu'aux pins des abords des plages languedociennes, pour placer cette exposition d'une très grande complexité sous le sceau de l'hybridité.

11 De Red, Green, Blue, Loom Music ${ }^{12}$ (2015-16), Starling déclare: " It found me » ${ }^{13}$ pour souligner l'importance de la rencontre dans son travail. L'installation est disposée dans trois pièces distinctes: le visiteur arrive devant un pianola faisant entendre un air joyeux. Dans une seconde pièce placée dans l'obscurité et séparée par un rideau, il voit un film tourné dans l'usine Antica Fabbrica Passamanerie Massia Vittorio datant de 1843 à Pianezza qui a la particularité d'utiliser d'anciens métiers à tisser fonctionnant, comme le pianola, avec des cartes perforées développées à l'époque par Joseph-Marie Jacquard (1752-1834) pour les métiers à tisser et utilisées dans les années 1840 par Charles Babbage pour concevoir l'ancêtre de l'ordinateur. C'est par ailleurs dans cette fabrique que Starling a découvert par hasard sur un piano la partition La Macchina Tessile, écrite en 2014 par le compositeur Rinaldo Belluci en hommage au son particulier des métiers à tisser. Starling a demandé au spécialiste du pianola Rex Lawson d'adapter la musique de Belluci pour cet instrument. La partition a quant à elle été convertie en image grâce à un logiciel de visualisation sonore ayant transformé la musique en bandes de couleur basées sur la fréquence et l'intensité des notes. Rendue visible, la partition a été ensuite traduite en cartes perforées permettant de créer une pièce textile de fils rouges, verts et bleus que l'on peut voir dans un troisième recoin de la troisième pièce, en face de la partition encadrée et à côté des cartes perforées.

12 Le morceau de musique original se voit donc transposé en pièce pour pianola, puis traduit en image grâce à un logiciel transformant la fréquence et l'intensité en couleurs, avant d'être transformé en carte perforée et en morceau tissé. La transformation de la partition en textile est filmée par l'artiste, puis diffusée par un vidéoprojecteur spécifique, dit CRT, utilisant des filtres rouge, vert et bleu reproduisant l'illusion de la quadrichromie et projeté dans la pièce obscure jouxtant le pianola, comme un écho au tissage présenté dans le film et comme la transposition visuelle incarnée d'un métier à tisser. Le film mime ce qui se passe dans l'atelier de tissage tout en renvoyant aux débuts de la photographie en couleurs. 

La bande-son du film fait écho à la partition de Belluci/Lawson, mais la musique qui s'écrit sur des feuilles de papier (partition transcrite et partition originale) se visualise également par le textile dans une surprenante synesthésie tactilo-visuelle. La musique, ou plutôt les musiques, se réalisent respectivement dans un instrument qui a la particularité de pouvoir être joué sans l'aide d'un musicien, le visiteur voyant les touches frappées comme par magie puisque la partition est inscrite sur un rouleau cylindrique perforé ; elles se réalisent dans des métiers à tisser, des cartes perforées, de l'étoffe tissée. La musique est celle des instruments (pianola et métiers), celle que voit le visiteur, celle qu'il entend, touche au point de l'illusion lorsqu'il regarde les effets du vidéoprojecteur. Le visiteur est ainsi confronté à une série de traductions différentes de la musique : le son du pianola, celui des métiers à tisser, celui de la carte perforée, de l'étoffe tissée, l'image de la feuille sur laquelle est imprimée la transcription de la partition et celle de la partition originale. Une même chose, l'immatériel des notes, se voit incarnée et matérialisée sous de multiples formes et médias (visuel, sonore et tactile), créant un jeu complexe de différences et d'identité, destiné à faire réfléchir au monde sensible et aux conditions d'apparition des objets dans le monde ainsi qu'à leur nature.

L'installation suivante exprime l'intérêt de Starling pour les récits. ${ }^{14}$ At Twilight ${ }^{15} \mathrm{se}$ présente comme la trace d'une représentation théâtrale inspirée de At The Hawk's Well de W. B. Yeats, pièce jouée en privé en 1916, inspirée par Pound lui-même inspiré par le théâtre Nô, et d'un séjour au Japon de Starling pour son Project for a Masquerade (2011).16 Ce projet a demandé trois années et a donné lieu à deux expositions à Glasgow et à trois représentations ${ }^{17}$ en plein air à Holmwood House en août 2016 : At Twilight est donc bien la trace d'une représentation de la réécriture de la pièce de Yeats.

L'installation s'expérimente dans une pièce plongée dans l'obscurité où apparaissent sur des troncs calcinés destinés à servir de support les masques des personnages créés par Yeats et revisités par Starling et l'artiste Yasuo Miichi : Cuchulain, naguère joué par Pound, le Vieil homme, gardien du puits (qui était une femme chez Yeats), le chorégraphe japonais Michio Itō qui a travaillé avec Yeats pour créer la figure de la femme-épervier. Masques auxquels s'ajoutent celui de Pound, celui du Rock Drill de Jacob Epstein (œuvre détruite par l'artiste), celui de Nancy Cunard, muse et amante de Pound, le tout accompagné d'une sculpture grandeur nature en deux parties du Bourriquet d'A.A. Milne, qui semble avoir perdu sa mélancolie originaire. Starling propose une audacieuse réécriture-hommage à une part du Modernisme ou plutôt à la trace d'une part du Modernisme transatlantique.

L'installation est encadrée par un dessin de l'épervier tiré du dessin original de Dulac, à une extrémité de la pièce, et par une vidéo représentant la danse de l'épervier tirée de la pièce de Starling et Graham Eatough, mettant en scène un danseur portant une reproduction du costume d'origine. Les troncs, les masques inspirés des très beaux masques du théâtre Nô et réalisés par un artiste japonais, sont ainsi disposés pour former un espace quasi théâtral, encadré par la vidéo montrant les mouvements lents et stylisés du Nô et par la représentation de l'épervier. L'installation est à la fois reviviscence et réinterprétation d'une pièce fonctionnant sur l'hybridité entre le théatre japonais et irlandais. Starling ne vise pas l'impossible reproduction à l'identique : il joue sur l'écart entre l'original à jamais perdu, n'existant que sous forme de traces, et la répétition/adaptation/réécriture. Son installation est à la fois 
hiératique, dépouillée et complexe ; elle est fort détaillée grâce à un véritable amour et respect du matériau et de la tradition évocateurs du travail de William Morris. De nouveau, le visiteur fait l'expérience d'une série de métamorphoses (ce que Starling appelle des "traductions») d'une pièce fonctionnant sur la métamorphose et l'hybridation en une autre, tandis que les masques, destinés à matérialiser l'identité chez un Yeats empreint de symbolisme européen se font écho en un étrange dialogue sans paroles. L'installation procède à une complexe hybridation de l'ancien (moderniste !) et du contemporain tout en rendant un hommage aux techniques dramatiques japonaises.

L'installation suivante, moins complexe, est peut-être plus émouvante car elle met en jeu la reviviscence corporelle du passé. $E l ~ E c 0^{18}$ (2014) est une installation vidéo née de la fresque murale du sculpteur Henry Moore, source d'inspiration constante pour Starling, qui fut à l'origine, en 1953, d'une fresque monumentale au Museo Experimental El Eco dont il ne reste que peu de traces, sauf quelques photographies en noir et blanc. Hommage à l'art mexicain, peuplée de squelettes, la fresque avait été réalisée pour l'inauguration du Musée de Mexico City qui avait en outre vu danser une jeune danseuse de quinze ans, Pilar Pellicer. C'est cette même artiste que Starling a filmée en 2014 au Musée le jour de la fête des morts. Devenue star de telenovelas, Pilar Pellicer a accepté de refaire les mouvements chorégraphiques de 1953 tandis que Starling la filmait. Son installation vidéo mêle les images de 1953 et celle de la performance de 2014 tandis que la bande-son de Joshua Abrams renvoie aux chansons populaires mexicaines entrecoupées par le son du clic-clac de l'obturateur photo. L'installation propose un va-et-vient entre passé et présent, corps adolescent et corps vieillissant, traces et œuvre contemporaine, Moore et Starling unis à travers les époques par le corps de Pilar Pellicer. La mise en scène de la mémoire des gestes et du corps par la danseuse offre une émouvante méditation sur les conditions de réalisation de l'art.

L'exposition s'achève sur The Liminal Trio Plays the Golden Door ${ }^{19}$ (2017), installation représentant la rencontre imaginaire de trois musiciens arrivant d'Europe à Ellis Island au début $\mathrm{du} \mathrm{xx}^{\mathrm{e}}$ siècle. Starling l'a conçue à partir de discussions récentes sur la politique migratoire trumpienne et d'un fonds d'archives d'un des administrateurs d'Ellis Island, Augustus Frederick Sherman (1865-1925), photographe amateur passionné ayant pris plus de 250 clichés d'immigrants arrivant dans leurs costumes traditionnels entre 1892 et 1925. L'artiste a ainsi fait reproduire les photographies de trois personnes venant respectivement de Hollande, d'Italie et de Roumanie et portant chacun le costume et l'instrument de leur pays. Ces portraits organisent et clôturent la salle d'exposition en se répondant sur trois murs. Leur structure ternaire évoque certes une scène mais délimite également l'espace d'une cérémonie intime et paradoxalement publique de re-collection. Ces clichés de taille respectable encadrent ainsi des supports métalliques simples, vêtus partiellement de costumes similaires à ceux des immigrants et portant chacun la réplique exacte de l'instrument original : la zampogna (ancêtre de la cornemuse), le caval (flûte typique des Balkans) et les sabots hollandais de Vollendam.

La bande-son a été enregistrée lors d'une session de musique organisée par Starling à New York avec trois musiciens pour créer un air où dialoguent les trois instruments et les traditions qu'ils expriment. Le morceau est ainsi diffusé via trois haut-parleurs placés aux côtés des trois mannequins. En dépit de son dépouillement apparent renforcé par le noir des supports et par le camaïeu de gris des costumes hérité des 
clichés originaux, il s'agit donc d'une installation extrêmement complexe puisque les figures des absents sont matériellement dissociées entre le son des instruments, l'image photographique et le support partiellement costumé. La dimension visuelle se diffracte entre les photographies d'archives, comme le sont les objets entre les répliques des instruments et les répliques exactes des costumes réalisées par Gustavo Gonzalez. Cette dissociation entre son, image et objet montre une identité fracturée, subtil pied de nez à la politique de l'identité qui finit immanquablement en politique identitaire discriminatoire et xénophobe. Le sujet, car c'est bien sur lui que médite cette fois l'artiste, est diffracté entre son, support, costume, représentation photographique, célébrant sa disparition au profit de l'avènement de sa présence. Ce sont ces étranges et émouvantes présences, étranges dans un univers pensant presque exclusivement en termes d'identités, que Starling convoque, évoque et fait cohabiter spatialement, musicalement, visuellement, tactilement. Cette assemblée des présences, cette liminalité faite œuvre, convoque la mémoire de l'émigration, de l'exil transformant la cérémonie intime en expérience publique tout en invitant le spectateur à faire l'expérience de sa propre mémoire, de son propre exil et de sa propre présence via le détour par ces trois absents.

Refusant la politique identitaire au profit d'une réflexion sur l'identité de l'objet (qui nie toute prétention à l'adequatio rei et verba) et sur la présence (toujours sur fond d'absence et d'exil), Starling propose une exposition complexe, moins empreinte d'humour que de gravité qui fait dialoguer ses quatre installations (la temporalité de leur création et les temporalités que chacune déploie, entre passé, présent et avenir de la rencontre avec le public) pour se placer sous le signe de la musique : musique du pianola, de la zampogna et du caval, des sabots, musique du théâtre Nô-Irlandais, qui toutes témoignent du soin apporté par l'artiste à la dimension sonore de son art. ${ }^{20}$ Multimédia ou plutôt " multi-layered $»^{21}$, son exposition propose non des hybrides, mais une pluralité de traductions d'une forme artistique en une autre, en plusieurs autres, avec, à chaque fois, une matérialisation travaillée comme un objet d'art destinée à la réflexion du visiteur sur l'identité intrinsèque qui ne se conçoit qu'à travers la différence elle-même créatrice de la causalité, de la temporalité et de la prae-sens.

\section{NOTES}

1. http://mrac.laregion.fr/exposition_fiche_mrac/222/3117-expositions-art-contemporain-avenir.htm

Je remercie Isabelle Durand, médiatrice au Musée d'art contemporain, de m'avoir invitée à faire une visite de cette exposition nourrie de la documentation qu'elle m'a par ailleurs envoyée.

2. Voir ses œuvres sur https://www.artsy.net/artist/simon-starling/works

3. Philip Kaiser, «Interview with Simon Starling, Berlin, March 2005 », Cuttings, np.

4. Philip Kaiser, «Interview with Simon Starling, Berlin, March 2005 », Cuttings, np.

5. Voir Raphaël Brunel, "Simon Starling, Thereherethenthere", 02 revue d'art contemporain trimestrielle et gratuite hiver 2009/2010 n52, p. 10-15. 
6. Le double de la pierre est exposé sur un socle construit selon les mêmes dimensions que celle de la plate-forme ayant servi à convoyer la pierre originale via la marée.

7. Three White Desks (2009) a pour point de départ le bureau créé par Francis Bacon pour Patrick White qui s'en sépara et le regretta au point de le faire reproduire par un ébéniste de Sydney avec un résultat décevant. Ayant retrouvé la photo dudit bureau, Starling la scanne et l'envoie à un ébéniste berlinois qui refait le bureau. Starling le photographie et envoie la photo à un ébéniste australien qui fait à son tour un bureau et envoie le résultat sous forme d'image numérique à un ébéniste londonien. Trois bureaux très différents sont ainsi obtenus, l'artiste s'offrant une réflexion sur l'unique et le différent en art, et sur le geste artisanal.

8. Il s'agit du premier ordinateur conçu en 1936 par Suze dans l'appartement familial, programmé à l'aide de bandes perforées entrées dans un lecteur. Suze perforait ses programmes dans un film photo $35 \mathrm{~mm}$. Starling crée une séquence animée de 30 secondes montrant le lecteur du film perforé qui a demandé 3992837240 octets soit 22 millions de fois la mémoire du Z1. Puis cette reconstruction informatique virtuelle est transférée sur un film $35 \mathrm{~mm}$ et projetée avec un projecteur de cinéma du modèle Dresden D1.

9. Il s'agit de deux épreuves contemporaines au platine à partir d'une photo du pavillon des innovations techniques allemandes de l'exposition universelle de Barcelone en 1929. Les épreuves sont illuminées à l'aide d'un projecteur alimenté par une pile à combustible fonctionnant par une réaction hydrogène/oxygène sur un catalyseur en platine, le tout séparé par un écran vertical en verre fumé légèrement incurvé.

10. Sculpture en bronze d'une particule d'argent créée à partir d'une épreuve photographique originale au gélatino-bromure d'argent de Moore intitulée Figure allongée $n^{\circ} 4$ (1955) agrandie 300 000 fois.

11. Starling choisit huit pierres pour former l'une des extrémités d'un mur de 6 mètres de long ; leur forme est ensuite scannée et reproduite de sorte à pouvoir créer leur reflet exact à l'aide d'outils de taille de pierre de pointe.

12. Red, Green, Blue, Loom 2015-16.

Weber Duo-Art pianola, carte perforée, rideau de ls, écran de projection, matériel audio, vidéoprojecteur CRT, socle, HD vidéo en boucle. Film $13 \mathrm{~min} .6 \mathrm{sec}$./carte perforée, $3 \mathrm{~min}$. $16 \mathrm{sec}$. Photographe Sebastiano Pellion di Persano. Courtesy de l'artiste et de la Galleria Franco Noero, Turin.

13. Entretien du 3 novembre 2017 avec l'auteur.

14. Simon Starling, «I plunder narrative structures from all over the place.» Philip Kaiser, « Interview with Simon Starling, Berlin, March 2005 », Cuttings, np.

15. At Twilight: W.B. Yeats, 2014-2016.

8 masques, 4 costumes, 1 drap, 9 arbres en bois carbonisé, lutrin, crochets muraux, 3 socles, 2 fleurets d'escrime, HD projection vidéo, texte mural. Dimensions variables. Courtesy de l'artiste et The Modern Institute/Toby Webster Ltd., Glasgow. Commande: The Common Guild, Glasgow.

16. Project for a Masquerade, 2011: 9 personnages iconiques de la guerre froide, un film et une proposition de représentation théâtrale incorporant l'histoire d'une œuvre de Moore existant elle-même sous différents aspects et noms comme Nuclear Energy (1967) grand monument à Chicago et Atom Piece (1964-5) petite sculpture de bronze acquise par le musée d'Hiroshima en 1987.

17. At Twilight/A Play for Two Actors, Three Musicians, One Dancer, Eight Masks (and a Donkey Costume). Mise en scène à Holmwood House, Glasgow, 2016.

18. El Eco, 2014. Film couleur $35 \mathrm{~mm}$ avec son transféré en HD, mur de plâtre, $11 \mathrm{mn} 18 \mathrm{~s}$. Courtesy de l'artiste et de la galerie neugerriemschneider, Berlin.

19. The Liminal Trio plays the Golden Door, 2017. 3 photographies argentiques sur gélatine type LE / virées au sélénium, 3 répliques de costumes, zampogna, kaval, sabots, 3 supports, 3 hautparleurs, 1 fichier audio. Dimensions variables. Courtesy de l'artiste et Kasey Kaplan, New York. 
20. Starling a travaillé avec des compositeurs et des musiciens : Joshua Abrams et son groupe le Natural Information Society, le pianiste Rinaldo Belluci, Rex Lawson qui a repris la composition de Belluci pour le pianola, le joueur de caval Winne Clement, de zampogna Sean Folsom, et la danseuse de sabots Livea Vanaver.

21. Entretien de Simon Starling avec l'auteur le 03/11/2017.

INDEX

Mots-clés : Simon Starling, exposition, art contemporain, multimédia, hybridité, transmédialité Keywords : Simon Starling, exhibition, contemporary art, multimedia, hybridity, transmediality Thèmes : British painting

\section{AUTEURS}

\section{BÉNÉDICTE COSTE}

Professeur d'études victoriennes

Université de Bourgogne Franche-Comté

bncoste@free.fr 